\title{
Estimation of carbon abundances in metal-deficient stars. Application to the "strong G-Band" stars of Beers, Preston, \& Schectman
}

\author{
Silvia Rossi ${ }^{1}$, Timothy C. Beers ${ }^{2}, \&$ Chris Sneden ${ }^{3}$ \\ ${ }^{1}$ IAG, Department of Astronomy, Universidade de São Paulo, \\ São Paulo, SP 05508-900, Brazil \\ email: rossi@astro.iag.usp.br \\ ${ }^{2}$ Department of Physics \& Astronomy and JINA: Joint Institute for Nuclear Astrophysics, \\ Michigan State University, E. Lansing, MI 48824 \\ email: beers@pa.msu.edu \\ ${ }^{3}$ Department of Astronomy, University of Texas, Austin, TX 78712 \\ email: chris@verdi.as.utexas.edu
}

\begin{abstract}
We develop and test a method for the estimation of carbon abundance ratios, [C/Fe], and metallicities, $[\mathrm{Fe} / \mathrm{H}]$, in metal-deficient stars, based on application of artificial neural networks and synthesis models to medium-resolution (1-2 $\AA$ ) spectra in the region of the $\mathrm{CH}$ Gband feature at $\lambda 4300 \AA$. We calibrate this method by comparison to modern carbon abundance determinations for $\mathrm{N} \simeq 125$ stars reported in the recent literature. As a first application, we estimate the abundance of carbon and $[\mathrm{Fe} / \mathrm{H}]$ for the sample of 56 stars identified as carbon-rich, relative to stars of similar metal abundance, in the sample of "strong G-band" stars discussed by Beers, Preston, \& Shectman.
\end{abstract}

Keywords. Stars: abundances, stars: carbon, stars: Population II, techniques: spectroscopic

The ongoing medium-resolution spectroscopic follow-up of candidate low-metallicity stars selected in the HK survey (Beers, Preston, \& Shectman 1992, BPSII; Beers 1999) and in the Hamburg/ESO Survey (HES; Christlieb 2003) has discovered large numbers of stars with carbon enhancements in excess of $[\mathrm{C} / \mathrm{Fe}] \simeq+1.0$. In order to quantify and understand the varieties of mechanisms for explaining the origin of CEMP stars it is necessary to develop procedures by which $[\mathrm{Fe} / \mathrm{H}]$ and $[\mathrm{C} / \mathrm{Fe}]$ measurements may be rapidly obtained for as large a number of stars as possible. Ideally, this should be based on medium-resolution (1-2 $\AA$ ) spectroscopy, since this information is already available for many thousands of metal-poor stars from the HK and HES follow-up campaigns, and will be available for hundreds of thousands of stars as new-generation surveys proceed.

We have investigated three alternative techniques for obtaining carbon abundance estimates of metal-deficient stars: an Artificial Neural Network (ANN) approach, a regression approach, and spectral synthesis. For all of these exercises, we require independent knowledge of $[\mathrm{Fe} / \mathrm{H}]$ and $[\mathrm{C} / \mathrm{Fe}]$, which we obtain primarily from the recent literature.

Using the ANN approach, we seek to estimate $[\mathrm{Fe} / \mathrm{H}]$ from the $\mathrm{CaII} \mathrm{K} K P$ indices and $(J-K)_{0}$, and $[\mathrm{C} / \mathrm{Fe}]$ based on two alternative sets of inputs: $(1) K P, G P$, and $(J-K)_{0}$, and (2) from the $K P$ and $G P$ indices alone.

We have also explored the use of a multiple regression approach, similar to that used by Beers et al. (1999) for the prediction of $[\mathrm{Fe} / \mathrm{H}]$. Our preference is to make use of ANN's, because one can train a suitable network very quickly, the technique allows for non-linear 

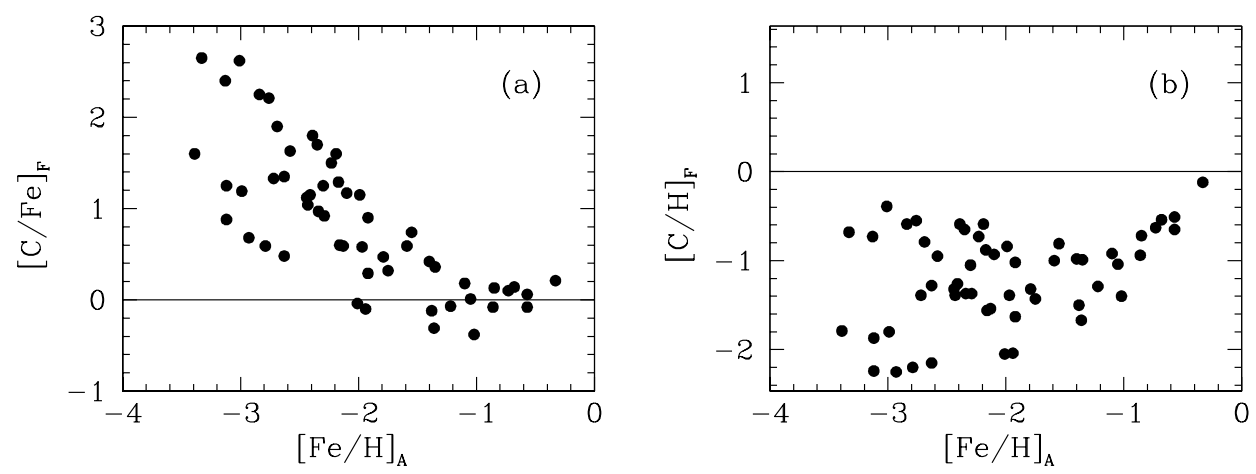

Figure 1. Final estimates of the carbon ratios (a) $[\mathrm{C} / \mathrm{Fe}]_{\mathrm{F}}$ and $(\mathrm{b})[\mathrm{C} / \mathrm{H}]_{\mathrm{F}}$, as a function of the ANN estimate of metallicity, $[\mathrm{Fe} / \mathrm{H}]_{\mathrm{A}}$, respectively

interactions of the predictor variables over the parameter space, and as new (or improved) calibration data come available, the ANN approach allows for rapid retraining.

Using these methods we are able to reproduce the previously-measured carbon abundances and $[\mathrm{Fe} / \mathrm{H}]$ determinations with an accuracy of $\simeq 0.25$ dex for stars in the metallicity interval $-5.5 \leqslant[\mathrm{Fe} / \mathrm{H}] \leqslant-1.0$, and with near-IR colors $0.2 \leqslant(J-K)_{0} \leqslant 0.8$.

For the third approach, we computed synthetic spectra of calibration and program stars over essentially the entire wavelength range of the CH G-band, $4190 \AA \leqslant \lambda \leqslant 4425 \AA$. The current version of the LTE line analysis code MOOG (Sneden 1973) was used to generate these spectra. For stars that are either very cool, or have very large $[\mathrm{C} / \mathrm{Fe}]$, the synthesis approach is to be preferred, because of the contamination of the sidebands of the GP index by molecular carbon lines (for more details see, Rossi et al. 2005).

Figures $1(\mathrm{a})$ and $1(\mathrm{~b})$ show the adopted estimates of $[\mathrm{C} / \mathrm{Fe}]$ and $[\mathrm{C} / \mathrm{H}]$ vs. $[\mathrm{Fe} / \mathrm{H}]$, respectively, for the "strong G-band" stars of BPSII. Note that many stars with $[\mathrm{Fe} / \mathrm{H}]$ $>-1.0$ are not in fact carbon-enhanced, although they were flagged as possible cases in BPSII. The envelope of $[\mathrm{C} / \mathrm{Fe}]$ clearly becomes more extreme for the stars with lowest $[\mathrm{Fe} / \mathrm{H}]$. However, as is seen in Figure $1(\mathrm{~b})$, the upper envelope of $[\mathrm{C} / \mathrm{H}]$, even at low $[\mathrm{Fe} / \mathrm{H}]$, reaches a maximum value of $[\mathrm{C} / \mathrm{H}] \approx-0.5$. This level of enrichment, about a factor of three less than solar, provides a constraint on the possible mechanisms that are responsible for the enrichment of carbon in the early Universe.

\section{Acknowledgements}

S.R. acknowledges partial support from the Brazilian agency FAPESP. T.C.B. acknowledges partial support of this work from grants AST 95-29454, AST00-98508, AST 00-98548, AST 04-06784, and PHY 02-16783, Physics Frontier Centers/JINA: Joint Institute for Nuclear Astrophysics, awarded by the U.S. National Science Foundation. C.S. acknowledges partial support from U.S. NSF grant AST 03-07495.

\section{References}

Beers, T.C., Preston, G.W., \& Shectman, S.A. 1992, AJ 103, 1987 (BPSII)

Beers, T.C., Rossi, S., Norris, J.E., Ryan, S.G., \& Shefler, T. 1999, AJ 117, 981

Beers, T.C. 1999, in Third Stromlo Symposium: The Galactic Halo, eds. B. Gibson,T. Axelrod, \& M. Putman (ASP: San Francisco), 165, p. 206

Christlieb, N. 2003, Reviews in Modern Astronomy 16, (Wiley: New York), p.191

Rossi, S., Beers, T.C., Sneden, C., et al. 2005 AJ, in press

Sneden, C. 1973, ApJ 184, 839 